\title{
Editorial
}

\author{
Bill Newsom
}

doi:10.3396/ijic.V4s1.014.08

IFIC is very pleased to present these papers from the Oxoid Infection Control Prize competition as a supplement to the IFIC Journal. The prize, which is presented annually, is for the 'Infection Control Team of the year'. Prizes of $£ 5000, £ 1000$ and $£ 500$ are awarded. To enter, you have to summarise the infection control challenges faced in your hospital, the communication methods used to respond to these challenges, the work undertaken, outcomes and improvements made, and finally say why you think your team should win this prestigious award. It is open to healthcare workers in any country of the world. The judges have included representatives from IFIC, The Hospital infection Society, The Infection Prevention Association, and eminent infection control professionals.

Oxoid is a major supplier of microbiological culture media and accessories worldwide. For many years Eric Bridson was its technical consultant and I am indebted to him for this short history:

Medical microbiology really starts with Robert Koch, working in Berlin in the 1880's. He was the first to grow bacteria reliably on solid surfaces, so allowing colonies to be seen and identified. Originally he used a meat extract with gelatine, however this dissolved at room temperature, and it was Fanny Hesse (Figure 1), wife of one of Koch's co-workers who suggested using agar. This had been used by her for making jellies, a trick learned from Indonesia. Meat extract was available in Germany thanks to Justus Von Liebig, but it was expensive. So a large scale manufacture was set up in Uruguay by the Liebig's Extract of Meat Company, which was registered in the UK. The product was called LEMCO (work it out). LEMCO was primarily used for drinks (OXO still is available in the UK). By 1910 a German Company - E. Merk was producing dehydrated media for growing bacteria, and was soon joined by DIFCO, who produced nutrient agar in the USA in 1916. Bacteriology in the UK only really

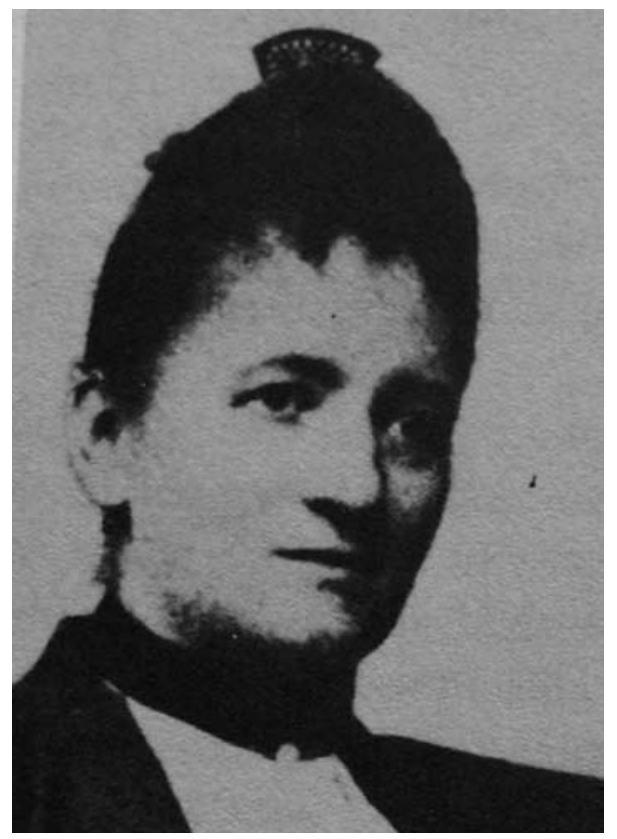

Figure 1. Fanny Hesse 


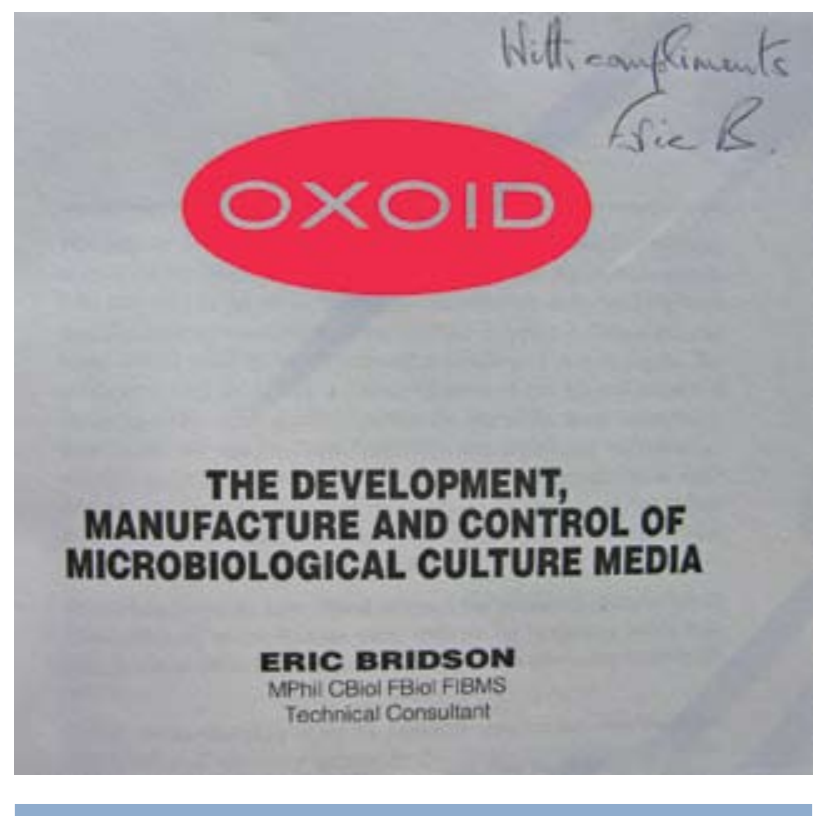

Figure 2. An Oxoid publication

took off after the war ended in 1945. Use of DIFCO media in the UK at that time was limited by cost and availability, and demands were unmet. Oxoid, already the medical branch of the Liebig's Company, decided to manufacture laboratory products in the UK in the 1950's. It was already selling LAB-LEMCO which was the basis of many of the culture media, which I used in my early days in the laboratory.

Oxoid has always had a good two-way relationship with its customers. I well remember the 'Oxoid Manual' describing culture media and their use. As more species of bacteria were discovered (for example legionella or campylobacter) - so the range of media expanded, and by 1999 the manual was in its eighth edition. Eric Bridson also produced a 'vade-mecum of microbiology' as well as a book on the history of culture media (Figure 2). Next year Oxoid celebrates thirty years of its journal 'Culture', which is distributed free to 20000 microbiologists worldwide. This has covered many topics over the years - including infection control, with an article on Semmelweis. Its 30th anniversary issue will be devoted to 'Hospitalacquired Infection'. Thus the Oxoid Infection Control Prize fits very well with the Company spirit.

The seven papers printed here come from around the world: Cameroon, Thailand, Egypt, England, and Northern Ireland, countries with varied challenges and very different availability of resources. The one common property of all the authors and their teams is - motivation. The main resource they have all used is time. Time to study just what was happening in their hospital, and to audit standards of hygiene and patient protection. Time to meet, agree, and lay down policies and to educate their healthcare staff.

Many of the common problems are described - the approach to AIDS and blood borne infection, the dangers of needle stick injuries, ventilator associated pneumonia, the superbugs - MRSA and Clostridium difficile, the need for hand hygiene, and for cleaning. Close co-operation with the hospital pharmacy to develop stewardship of antibiotic prescribing is another strategy that may well save money - both on drugs, and on patient care. This could be on various levels - I was indeed chairman of both the infection control and pharmacy committees in Cambridge, but perhaps a more down to earth approach is to have a pharmacist on the infection control team.

Once policies have been laid down - there is the need to educate staff in their use, and later to audit the outcome. One ingenious low-cost suggestion is use of computer 'screen savers' to display infection control messages instead of pictures of mountains or the ocean etc.

Hand hygiene is mentioned by most authors, and today many publications are concerned with facilities, what agent to use, staff education, and audit of their hand care. I was first taught surgical hand disinfection

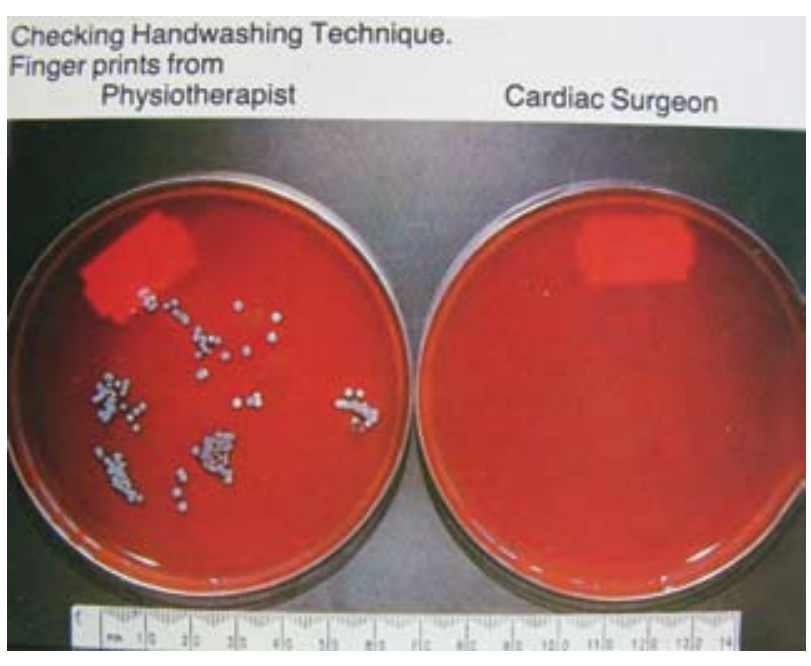

Figure 3. Blood agar plated showing finger prints after hand disinfection 
by my father, who was a surgeon. He scrubbed each hand for five minutes with soap and water (remember Bill each finger has four sides). Probably not a good thing, but he then retrieved the situation by dipping his hands in alcohol before putting his gloves on.

Many methods of monitoring hand hygiene have been described. The least invasive was use of a video camera over the sink. However this must have required a lot of time to analyse the film! My own approach, for the strictly limited situation of a single room in which the first heart transplant patients were nursed, was to start by having a pile of blood agar plates by the sink, and ask each person to make an impression of their fingertips on the plate after washing. The results are seen in Figure 3. The plates were shown to staff the next day - and this made them very aware of the problem!

Many of the main principles of infection control are described in these papers in a clear way. Many require more common sense than money. They serve as a good introduction to the IFIC 'Basic Concepts of Infection Control' manual, which provides answers in greater detail.

\section{Note from IJIC's Editor-in-Chief}

IFIC is grateful to Oxoid for their support, and for allowing us to publish these papers. Together, they represent the best in world wide experiences in developing infection control.

The Oxoid prize is a yearly event, and as evidenced by the submissions printed in this supplement, it is not only the winners who demonstrate they can make a significant contribution to Infection prevention and control in their countries.

We hope these articles will encourage all of our readers, wherever in the world, to have a go!

Judith Richards 